\title{
Estrategias de enseñanza y el uso de las tecnologías de información y comunicación en las instituciones educativas departamentales en el Municipio Zona Bananera - Colombia
Teaching strategies and the use of information and communication technologies in departmental educational institutions in the Bananera Zone Municipality - Colombia

\author{
Luis Mariano Urueta Vélez \\ luismariano71119@gmail.com \\ Instituto Educativo Departamental Telma Rosa Arévalo \\ Departamento de Magdalena \\ Colombia \\ https://orcid.org/0000-0003-2278-2448
}

Recibido: 16 de octubre del 2018

Aprobado: 01 de diciembre del 2018

\begin{abstract}
RESUMEN
La finalidad fue Determinar la relación entre las Estrategias de enseñanza y el uso de las Tecnologías de Información y Comunicación en las Instituciones Educativas Departamentales en el municipio Zona Bananera - Colombia. Se respaldó en los postulados teóricos de Marín, (2010), Mosley, Megginson, y Pietri, (2005), Cuello (2009), entre otros; se enmarca en el paradigma positivista - cuantitativo, se cataloga como descriptiva correlacional, con un diseño de campo, transeccional y no experimental. La población estuvo constituida por (170) docentes. La recolección de la información, se utilizó la técnica de observación mediante encuesta, con un cuestionario conformado (60) reactivos, validado por expertos, con una prueba piloto a 10 sujetos, se le aplicó el coeficiente Alpha de Cronbach dando como resultado 0,96. Adicionalmente, se aplicó la fórmula del coeficiente de Pearson, dando como resultado fue de $r=0,490$. Para el análisis, se utilizó la estadística descriptiva, utilizando para el cierre de las variables Análisis de Varianza (ANOVA). Se pudo inferir que en las instituciones educativas en contexto, las estrategias de enseñanza y el uso de las TIC, no son aprovechadas por los docentes en el proceso educativo, dado que no se encuentran involucrados en la promoción de aprendizajes significativos a partir de los contenidos escolares.
\end{abstract}

Descriptores: Estrategias de enseñanza; uso de las TIC; educativas; aprendizaje; contenidos escolares. 


\section{ABSTRACT}

The purpose was to determine the relationship between the teaching strategies and the use of Information and Communication Technologies in the Departmental Educational Institutions in the municipality of Zona Bananera - Colombia. It was supported in the theoretical postulates of Marín, (2010), Mosley, Megginson, and Pietri, (2005), Cuello (2009), among others; it is framed in the positivist - quantitative paradigm, it is cataloged as descriptive correlational, with a field design, transectional and not experimental. The population was constituted by (170) teachers. The information was collected, the observation technique was used by means of a survey, with a questionnaire formed (60) reactive, validated by experts, with a pilot test to 10 subjects, the Alpha coefficient of Cronbach was applied, resulting in 0.96 . Additionally, the formula of the Pearson coefficient was applied, resulting in $r=0.490$. For the analysis, descriptive statistics was used, using for the closure of the variables Analysis of Variance (ANOVA). It could be inferred that in educational institutions in context, teaching strategies and the use of ICTs are not used by teachers in the educational process, given that they are not involved in the promotion of meaningful learning from the content school.

Descriptors: Teaching strategies; use of ICT; correlation; learning; school contents.

\section{INTRODUCCIÓN}

A nivel mundial la sociedad está sumergida en cambios que surgen en los escenarios tecnológicos organizacionales donde las naciones deberán organizar y adaptar modelos propuestos. En este sentido, se deben cumplir con las modificaciones como consecuencias de los cambios globales que son implementados por el sistema educativo, de esta forma, debido a estas transformaciones el personal que en ella labora deberán ser capacitados para impulsar el desarrollo del país.

Tomando en consideración que los docentes desempeñan una diversidad de funciones que sin duda en materia organizativa se requiere habilidades en el uso de las tecnologías a fin de dar respuestas a las exigencias del mercado de trabajo actual. En el mismo orden de ideas, la aplicación de la tecnología va más allá de los procesos individuales, comerciales y cotidianos, ha revolucionado la forma de actuar de las personas transformando de manera rápida su entorno vital y aplicado para mejorar la calidad de vida. 
Sin embargo, se ha considerado a las escuelas como un elemento más de la sociedad, encargada de proporcionar conocimiento, descubrimiento y nuevos puntos de vista sobre el mundo actual, donde se ve obligada a la aplicación. Asimismo, los recursos se deben utilizar con mayor eficacia debida a las limitaciones financieras, la planificación estratégica y la gestión de las Tecnologías de la Información y Comunicación adquieren una importancia especial para cumplir con los objetivos de eficiencia impuestos por la organización.

De igual manera, es importante destacar que para alcanzar el objetivo que se ha trazado el gobierno Nacional de hacer de Colombia el país mejor educado de América Latina en 2025, fue necesario definir metas anuales que permitan un nivel de educación competitivo con relación a los estándares educativos de Latinoamérica.

Asimismo, el establecer estrategias de enseñanza para el uso de las TIC el brindar herramientas que favorecen en las instituciones que no cuentan con bibliotecas ni con material didácticos. Estas tecnologías le permiten fácil acceso a docentes y alumnos, además de mejorar el ambiente de aprendizaje y hacer más divertido las áreas tradicionales del currículo. Proyecto colaborativo la escuela que se quiere.

Resulta pertinente señalar que, las estrategias de enseñanza permiten la aplicación y uso de las TIC; estas según Ibarra (2007), la integran una serie de recursos "empleados como herramientas comunicacionales, informativos y audiovisuales, constituyéndose en instrumentos, soportes y canales para el tratamiento o acceso a la información. De este modo, constituye nuevos soportes y canales para dar forma, registrar, almacenar y definir contenidos informacionales mostrando las acciones laborales del gerente educativo.

Por tanto, es necesario que ante los requerimientos para una verdadera integración de las TIC deban estar incluidas en el proceso de restructuración del Proyecto educativo institucional de Colombia y en su subsiguiente implementación en las Instituciones Educativas Departamentales en el municipio de la Zona Bananera. En tal sentido, el 
uso de las TIC debe reflejarse en la forma en que los estudiantes son examinados y evaluados.

Además, son excelentes recursos para la evaluación de los aprendizajes, de igual manera, Acceso a desarrollo profesional para los directivos y docentes en la aplicación de las mismas, para dominar su uso y facilitar el aprendizaje entre pares y el intercambio de recursos.

En ese sentido y en relación a lo expuesto en párrafos anteriores surge la necesidad de realizar el presente estudio el cual tiene como fundamento determinar la relación entre las Estrategias de enseñanza y el uso de las Tecnologías de Información y Comunicación en las Institución Educativas Departamentales en el municipio Zona Bananera - Colombia, dado que el nuevo reto de hoy, ya no se trata que el educador sea un buen controlador de la información científica y metodológica sino, que debe buscar que los estudiantes sean agentes activos y participativos dentro del proceso educativo.

\section{ARGUMENTO TEÓRICO}

A los frutos de una mayor comprensión del presente artículo, se extendió un resumen del trabajo, que constituye su sustentación teórica y que se estructuró de la siguiente manera:

\section{Estrategias de enseñanza}

Las estrategias son conscientes e intencionales, dirigidas a un objetivo relacionado con los procesos formativos. Son consideradas procesos mediante los cuales se eligen, coordinan, aplican habilidades, que se vinculan con la enseñanza y el aprendizaje. Dependen de los objetivos y contenidos que se persigan, como refiere Carrasco (2008), todas las estrategias son válidas cuando sean aplicadas de modo activo propiciando el ejercicio de la reflexión y del espíritu crítico del alumno. 
Por su parte, la enseñanza de acuerdo con, Pérez (2009) es el proceso sistemático, dirigido, que facilita la integración del estudiante a la sociedad a la que pertenece, proveyéndolo de contenidos, procedimientos y actitudes que le permitan ser un aporte a su entorno. Además, indica que la enseñanza es un proceso de transformación, más que de acumulación de conocimientos.

En el mismo orden de ideas, los planteamientos de Anijovich y Mora (2009) hacen un análisis en referencia a los diversos conceptos que han surgido sobre las estrategias de enseñanza, destacando las estrategias de enseñanza han tenido múltiples definiciones que suelen prestarse a interpretaciones ambiguas. En algunos marcos teóricos y momentos históricos, se ha asociado el concepto de estrategias de enseñanza al de técnicas, entendidas como una serie de pasos por aplicar, una metodología mecánica, casi un algoritmo.

Dentro de ese marco, Tobón (2008) las estrategias de enseñanza son un conjunto de acciones que se proyectan y se ponen en marcha de forma ordenada para alcanzar un determinado propósito, con ellas los estudiantes aprenden a reflexionar sobre la construcción de significados, la responsabilidad en la estructura y el proceso para obtener los conocimientos.

Mientras que, Beltrán (2008 p. 207) considera "las estrategias de enseñanza son un conjunto de actividades ejercidas por el docente para facilitar la adquisición de conocimientos, en una situación particular de aprendizaje". Asimismo, Maldonado (2010) define las estrategias de enseñanza como el conjunto de instrucciones o prescripciones ordenadas para regular el desarrollo de un proceso de intercomunicaciones que provoque experiencias de aprendizaje en los estudiantes.

Al respecto, Campos (2010 p. 89) explica "las estrategias de enseñanza son aquellas utilizadas por el profesor para mediar, facilitar, promover, organizar aprendizajes". En el mismo orden de ideas, Pimienta (2012 pág. 3) refiere que las estrategias de enseñanza "son aquellos instrumentos de los que se vale el docente para contribuir a la 
implementación y desarrollo de competencias en los estudiantes, las cuales deben usarse de manera permanente en la secuencia didáctica de la práctica educativa Mientras que, Piedra (2008) considera las estrategias de enseñanza como mediaciones instrumentales que facilitan la concreción de aprendizajes, las construcciones de conocimientos. Por otra parte, Roa (2006) destaca las estrategias de enseñanza favorecen la superación de obstáculos, amplían el campo de interés del alumnado, con tácticas motivadoras donde se promuevan la resolución de problemas cotidianos, por ende, es fundamental que el docente pueda enfocar la instrucción de tal manera que pueda hacer un balance apropiado entre el contenido que debe transmitir y los procesos del pensamiento que son necesarios para dicho contenido.

Para, Camilloni (2008 p. 186) las estrategias de enseñanza "son las formas en que el docente crea una situación que permita al alumno desarrollar la actividad de aprendizaje, recomendándose planificar una para cada situación didáctica, su variedad depende de la creatividad del educador".

Por último, Díaz y Hernández (2010) expresan que las estrategias de enseñanza son los procedimientos o recursos utilizados en forma reflexiva y flexible por el agente de enseñanza para promover aprendizajes significativos, estas pueden convertirse en medios o recursos de ayuda pedagógica, además, representan un elemento fundamental del proceso de aprendizaje, puesto que de ella depende la orientación y operatividad del proceso e implica una interrelación constante con los demás elementos del diseño instruccional, como lo son: los objetivos, los contenidos, las características y conductas de entrada de los alumnos, los medios instruccionales y la evaluación.

\section{Uso de las tecnología de la información y comunicación}

Las TIC cada vez están cobrando mayor auge en los espacios educativos. Por sus beneficios y potencialidades, se requiere de su uso en casi cualquier actividad humana. De manera que en las instituciones educativas se ha potenciado su uso como herramienta en la gestión del director, pero también de los docentes y estudiantes. 
De este modo, se puede decir que las TIC, ofrecen una variada fuente de investigación, pues se puede mediante una computadora conectada a la red Internet navegar para obtener material acerca de los proyectos educativos, a través del correo electrónico, enviar formación al personal, archivar datos, entre otras aplicaciones más. Por lo tanto, se requiere de habilidades para poder adentrarse al mundo de las tecnologías, a fin de obtener las habilidades necesarias para su uso, ofrecer ayuda a los docentes, capacitarles y enfrentar los retos que, en materia educativa supone el conocimiento de los recursos tecnológicos.

De igual manera, Marín (2010), opina que la incorporación de las TIC a los centros educativos, en resumidas cuentas, es sentida como algo positivo si se acompaña de una formación a los docentes, entendiendo esta formación no sólo como el proceso de aprendizaje en el uso de un recurso, además de ello enseñarles cómo se pueden integrar en el desarrollo de su área de conocimiento.

Según se ha citado, la incorporación de los recursos tecnológicos, en especial las computadoras a las instituciones educativas, han favorecido la aplicación de otras formas de impartir las clases, realizar material didáctico, ser más interactivos a la hora de realizar las labores propias de la institución. Por eso, el director debe gestionar los aspectos que mejoren a la institución. Pero, se requiere de la formación de los docentes, para que se le dé el uso adecuado a los mismos, ya que usualmente el conocimiento está allí, pero, es preciso recibir la capacitación oportuna para saber explotar los conocimientos de una manera positiva.

En este sentido, en las instituciones educativas, se requiere de personal directivo que doten de facultades tecnológicas a los docentes, quienes son los que tienen la misión de educar. En el mismo orden de ideas, López (2012), opina que el directivo debe "actuar como un profesional actualizado respecto a las innovaciones educativas, científicas y tecnológicas. Por lo tanto, como profesional de la enseñanza, el directivo debe estar actualizado, emplear las tecnologías en su quehacer cotidiano, saber acerca de los adelantos en el orden científico, cultural, deportivo, y las TIC's le ayudarán a ello. 
Pues, si se investiga a través de las redes, se podrán asimilar diversidad de conocimientos que hasta ahora eran inalcanzables con el uso de los métodos tradicionales de la enseñanza, que eran basados fundamentalmente en el uso de libros, revistas y demás impreso, pero ahora, hay recursos digitales que están a la disposición de todos y que mantienen actualizados a los miembros de las escuelas.

Por otra parte, Mosley, Megginson, y Pietri, (2005), opinan que la gestión del director incluyen el rendimiento y la capacidad para supervisar de una manera efectiva los procesos, prácticas o técnicas específicas que se requieren para desempeñar determinadas tareas. En las organizaciones educativas, se necesita del personal docente que pueda asimilar el uso de las TIC e irlas incorporando en la supervisión, de manera que, se integren los recursos tecnológicos en la gestión director.

Esto, se logra si se capacita en su uso, a la vez que entrena a los docentes para que digitalicen la información, envía correos electrónicos, solicita a través de las redes, al mismo tiempo, en las instituciones educativas, almacenando los archivos en las computadoras, pues allí luego podrán realizar las correcciones que ameriten en cada año escolar.

Así también, mediante las TIC, el Coordinador académico puede solicitar a los docentes y estos presentarlas en formato digital, lo que significaría un avance en materia educativa, porque desde el punto de vista tecnológico se estaría avanzando en la aplicación de técnicas de trabajo más rápidas y dinámicas, así se cumple con las tareas en el tiempo oportuno.

A su vez, Carda y Lamosa (2007), opinan que "las funciones de asesoramiento pedagógico, coordinación, facilitación del clima social. Control, difusión de información, gestión y representación. Como puede verse, el rector educativo, cumple con varias funciones, dentro de las que destacan, asesorar a su equipo de docentes, coordinar las acciones pedagógicas, estimular un ambiente de trabajo sano, establecer redes de comunicación, crear un correo electrónico o una página web para la institución, así todos estarían informados de lo que pasa en la misma. 
Por otra parte, el rector educativo brinda asesoría a los docentes en todos los ámbitos de la vida laboral, coordina sus acciones, así como el difundir la información, gestiona los recursos necesarios para cumplir con las tareas propias de cada proyecto emprendido, además, representa al plantel en las actividades tanto internas como externas. Por lo que, se requiere de directores actualizados, que sepan coordinar las actividades institucionales, hábiles en el uso de las tecnologías.

En palabras del investigador el uso de las Tecnologías de la Información y Comunicación opina que la incorporación de las TIC a los centros educativos, en resumidas cuentas, es sentida como algo positivo, así que la gestión del director incluye el rendimiento y la capacidad para supervisar de una manera efectiva los procesos.

\section{La contribución de las tecnologías de la información y comunicación}

Las TIC, aportan diversidad de beneficios dentro de los que se pueden citar los siguientes. Mejora la comunicación, la satisfacción y son un soporte para la gestión del conocimiento. De acuerdo a esto, se puede explicar que son de gran ayuda en el proceso del director, también en la gestión que realizan los docentes. Según, González (2010), expresa que ayudan a la comunicación interna, al igual que el logro de la motivación para la satisfacción personal e integración, sistemas de tratamiento en los datos obtenidos por las evaluaciones del desempeño, soporte para la gestión del conocimiento.

Tal y como se ha planteado, las TIC promueven la comunicación en las organizaciones escolares, pues, tanto el director como los docentes pueden comunicarse en todo momento, aunque del plantel no esté físicamente en la institución, podrán enviar mensajes de texto, además, se pueden conectar a través de la red. Además, se debe lograr involucrarse más con sus colegas porque se mantienen comunicados, se ayudan mudamente, en el manejo de las herramientas, sobre todo porque pueden almacenar los datos y utilizarlos en cualquier momento. 
Por su parte, Tobón (2008), refiere que las TIC darán valor a los recursos disponibles y desplegarán un modelo de aprendizaje organizacional, que permita cambiar y consolidar la estructura cognitiva de la empresa. Es por eso que el uso de las Tecnologías de Información y Comunicación en educación cobra cada vez más importancia y se hace necesario que tanto el directivo como los docentes aprendan a utilizarla, ya sea por el uso que pueda darse al desempeñar sus funciones en el ámbito personal, se amplían las formas de comunicación con los demás.

De la misma manera López (2012), explica que es importante señalar que ninguna de las nuevas herramientas reemplaza al profesor, se requiere un docente conocedor de la asignatura que administra, pero con una formación simultánea que le permita desarrollar competencias a fin de usar las mejores combinaciones de estrategias dentro de la institución. Como puede observarse, las tecnologías educativas son muy importantes para el desarrollo del proceso, pero en ningún momento sustituirán a los docentes, pues la parte afectiva también importa, y es el director o quien promueve las interacciones sociales persona a persona al igual que como con otros integrantes del plantel.

\section{ARGUMENTOS METODOLÓGICOS}

De acuerdo con, Pelekais, Raspa y Pelekais (2011, p.47), el enfoque epistemológico "viene dado por el proceso de descubrir y comprender los puntos esenciales de un problema o negocio para tratarlo o resolverlo acertadamente, a través de grandes lineamientos o compromisos conceptuales a los que denomina paradigmas". En tal sentido, para responder al posicionamiento epistemológico se considera que la presente investigación se identifica con el enfoque positivista, el cual para Hurtado (2010), concede la primacía a los hechos antes que a las ideas, a las ciencias experimentales antes que a las prácticas y las leyes físicas antes que los postulados de la filosofía.

Así mismo, la investigación se ubica bajo un enfoque cuantitativo, entendiendo este 
como aquel en el que se recogen y analizan datos cuantitativos sobre las variables, estudiando su relación o asociación. Al respecto para Hernández y otros (2012, p.352), definen este enfoque "como aquel que utiliza la recolección y el análisis de datos para contestar preguntas de investigación y probar hipótesis establecidas previamente y confía en la medición numérica, el conteo y frecuentemente en el uso de la estadística para establecer con exactitud patrones de comportamiento de una población".

De igual manera, Pérez (2010) la epistemología sistematiza el modo de obtener el conocimiento en la investigación y constituye un estudio critico de los principios de las diversas ciencias, de su valor y objetividad, implicando una reflexión sobre el origen lógico de los procesamientos a través de los cuales se alcanza el contenido científico y se forman las distintas ciencias.

Es por ello que para el presente estudio, se asume el positivismo como enfoque epistemológico, el cual sostienen que todas las actividades del mundo deben ser estudiadas y comprendidas únicamente enmarcadas en un estricto análisis de los hechos, los cuales son verificados por la observación y la experiencia expresándose con recursos cuantitativos. Según, es posición el conocimiento científico surgido como producto de esta investigación corresponde a la afirmación positiva de las teorías de gestión del conocimiento productividad investigativa a través de la aplicación del método científico.

De acuerdo al propósito que se persigue la investigación se enmarco en la tipología descriptiva correlacional. Según, Hernández, Fernández y Baptista (2012, p. 60) señala a la descriptiva como la que busca "describir situaciones, eventos, es decir como es y cómo se manifiesta determinado fenómeno"; en tal sentido, la investigación es descriptiva, puesto que pretende describir el problema planteado Instituciones Educativas Departamentales en el municipio Zona Bananera- Colombia, con una serie de descripción de hechos tal y como suceden en la realidad, proporcionara una serie de propiedades o características específicas que le permiten ser sometido a constatación y análisis. 
En el mismo orden de ideas, Tamayo y Tamayo (2012), considera que el estudio descriptivo es aquel que comprende el registro, análisis e interpretación de la naturaleza actual, y la composición o procesos de los fenómenos, en donde el enfoque va dirigido a las conclusiones dominantes o sobre como una persona, grupo o cosa funcionan en el presente, la misma trabaja sobre realidades de hecho y su principal característica es presentar una interpretación correcta.

De igual modo puede señalarse que la investigación en cuestión se considera correlacional porque tiene como propósito medir el grado de relación existente entre dos variables en los mismos sujetos, es decir, investigar hasta qué punto las variables observadas en un factor, se corresponden con las variables en uno o más factores, basándose en coeficientes de correlación, según explica Hernández y otros (2012).

En ese sentido, la utilidad y propósito principal de los estudios correlaciónales es determinar cómo se pueden comportar un concepto o variable conociendo el de las otras variables relacionadas; en este caso estrategias de enseñanza y el uso de las tecnologías de información y comunicación. Atendiendo a lo expuesto, el método a utilizar para la investigación planteada es descriptivo correlacional, por buscar obtener información de los hechos en su estado real, así como determinar el grado de relación entre estrategias de enseñanza y el uso de las tecnologías de información y comunicación en las Institución Educativas Departamentales en el municipio Zona Bananera - Colombia.

El diseño de investigación en el marco referencial de acción del investigador, comprende las estrategias para alcanzar los objetivos. A tal efecto el control de las variables y elección de sujetos es no experimental, que de acuerdo a Hernández y otros (2012, p. 189) "es aquel que se realiza sin manipular deliberadamente las variables permite observar el fenómeno tal como se presentan en el contexto natural para después analizarlo". Al respecto las variables estrategia de enseñanza y uso de las tecnologías de información y comunicación Instituciones Educativas Departamentales en el municipio Zona Bananera- Colombia, serán abordadas sin realizar ningún tipo de 
manipulación que pueda modificar los sucesos tal cual se perciben.

De igual forma, Díaz (2009, p. 91) considera que un estudio no experimental no se construye ninguna realidad, por cuanto esta ha sucedido en ausencia del investigador. Es decir, es una sola investigación sistemática y emperica en las cual las variables independientes no pueden ser manipuladas intencionalmente pues el fenómeno que las implica ya ha ocurrido.

Por su dimensión temporal fue considerada como un diseño transeccional o transversal, ya que se limitará al estado de las variables en un determinado punto en el tiempo; en relación a esta dimensión Hernández y otros (2012, p. 123) señalan "se caracterizan por recolectar datos en un solo momento, en un tiempo único, su propósito es descubrir las variables y analizar su incidencia e interrelación en un momento dado".

Por otra parte, el diseño fue de campo, el investigador observa la realidad de los hechos donde se producen. Según Hurtado (2010, p. 84), considera que el estudio de campo es aquel donde se describe un fenómeno, cuya información es obtenida de fuentes vivas o directas en su ambiente natural, es decir, dentro del contexto particular al cual corresponden sin producir cambios o modificaciones de ninguna índole.

Durante el desarrollo de la investigación resultó importante el proceso de obtención de la información, la cual permitió responder a las interrogantes de estudio y los objetivos definidos para tales efectos, Arias (2012), indica que las técnicas de recolección de datos son las distintas formas o maneras de obtener información, resultando importante que los datos generados de esta sean válidos y confiables, debiendo ser caracterizados por su pertinencia y coherencia con el hecho de estudio.

En razón de lo antes señalado, para efectos de estudio, se empleó como técnica la fuente primaria, a través del uso de la encuesta. Para Hurtado (2010), la técnica constituye el procedimiento utilizado para recolectar la información, entre ellos la encuesta, la observación, entre otros. En este sentido, Méndez (2009), señala que la encuesta comprende un instrumento empleado para la observación constituido por preguntas cuyas respuestas se tabulan según la opinión del encuestado lo cual permitió 
el conocimiento y opiniones de los encuestados en relación con su objeto de investigación.

Para elaborar el instrumento de recolección de datos, se tomó en cuenta los objetivos planteados dentro del estudio, ya que representan los parámetros que permitieron medir las variables. Para lograr esto, se realizó una matriz de objetivos, en los cuales se mostró una relación coherente tanto del objetivo general como de los específicos, así como de las variables, dimensiones e indicadores, para cada indicador se diseñó Ítems o preguntas, para las variables de estudio.

Para la interpretación de los datos, se procederá a elaborar un baremo a fin de ubicar los resultados obtenidos en el mismo y analizarlos, con el propósito de darle sentido explicativo a éstos, se establecerá un baremo de conversión de las alternativas de respuestas en categorías.

Según Tamayo y Tamayo (2012), el procesamiento de datos comprende la etapa, en la cual una vez recopilados los datos por los instrumentos diseñados para tal fin, es necesario procesarlos, es decir, elaborarlos matemáticamente, ya que la cuantificación y su tratamiento estadístico permitió la emisión de conclusiones. En tal sentido una vez recolectados los datos deben codificarse a través de símbolos o números tal y como los señalan Hernández y otros (2012), con la finalidad de poder resumir la información obtenida a fin de prepararla para el análisis.

\section{RESULTADOS DE LA INVESTIGACIÓN}

Una vez realizado el análisis y discusión de los resultados, seguidamente se procede a formular las conclusiones del proceso investigativo se tiene que en estas instituciones educativas, los recursos asociados a las TIC no son utilizados eficientemente por los docentes, demostrando falta de experiencia en conocimiento y uso de los medios informáticos de que se dispone en las aulas, desfavoreciendo en los alumnos las actitudes como ayudar a los compañeros, intercambiar información relevante encontrada en Internet y poder resolver problemas a otros compañeros. 
En cuanto a la relación entre las estrategias de Enseñanza y uso de las Tecnologías de la Información y Comunicación. Se logró determinar que existe una influencia o relación positiva media entre ambas variables desde la perspectiva de los resultados obtenidos del cuestionario aplicado a los docentes, en otras palabras, a medida que incrementa medianamente la participación de los componentes de la variable Estrategias de Enseñanza, en esa misma proporción incrementará la participación de los componentes de la variable Uso de las TIC.

De esta manera, se llegó a la conclusión de que, en las instituciones educativa en contexto, las estrategias de enseñanza y el uso de las TIC, no son aprovechas por los docentes en el proceso educativo, dado que no se encuentran involucrados en la promoción de aprendizajes significativos a partir de los contenidos escolares; aun cuando en el primer caso, el énfasis se pone en el diseño, programación, elaboración y realización de los contenidos a aprender por vía oral o escrita (lo cual es tarea de un diseñador o de un docente), y en el segundo caso la responsabilidad recae en el alumno.

\section{REFERENCIAS CONSULTADAS}

1. Anijovich, R y Mora, S (2009) Estrategias de enseñanza. Otra mirada al quehacer en el aula. 1a ed. la reimp. - Buenos Aires: Aique Grupo Editor, 2010. 128 p.

2. Arias, F. (2012). El proyecto de Investigación. Introducción a la metodología científica. Editorial Episteme. 5ta Edición.

3. Beltrán, J (2008) Procesos, estrategias y técnicas de aprendizaje. Madrid: Síntesis.

4. Camilonni, A (2008). El saber didáctico. Buenos aires. Editorial Paidos.

5. Campos, Y (2010) Tipos de estrategias de enseñanza - aprendizaje. Campos del conocimiento.

Disponible

en: http://www.camposc.net/Orepositorio/libros/estrategias/m2TiposdeEstrategias.htm 
6. Carda, R. y Larrosa, F. (2007). La organización del Centro Educativo. 2da. Ed. Editorial Club Universitario. España.

7. Díaz Barriga, F. y Hernández, G. (2010) Estrategias docentes para un aprendizaje significativo. Una interpretación constructivista. 3o Ed. México: McGraw Hill.

8. Díaz, V (2009) Metodología de la investigación científica y bioestadística: para médicos, odontólogos y estudiantes de ciencias de la salud. RIL Editores, 585 páginas. https://books.google.co.ve/books?id=ZPVtPpdFdGMC\&dq=Díaz++considera+que +un+estudio+no+experimental+no+se+construye+ninguna+realidad.

9. Gonzales, D. (2010). Psicología de la Motivación. La Habana: Ciencias Médicas.

10.Hernández, R., Fernández, C. y Baptista, L. (2012). Metodología de la investigación. Edición 4ra.

11. Hurtado, I. (2010). Paradigmas y métodos de investigación en tiempos de cambio. Venezuela. Editorial CEC.

12. Ibarra, (2007). Estrategias didácticas utilizadas para la formación de estudiantes en licenciatura en educación de la misión Sucre, Cumana. Estado Sucre. (Tesis de oriente núcleo de sucre) recuperado de http://ri.bib.udo.edu.ve/bitstream/123456789/3762/1/tesis_mmyiy.pdf.

13. López, J. (2012). La práctica de la innovación educativa y nuestro conocimiento sobre ella. Revista de curriculum y formación del profesorado, vol.14, nำ1.

14. Maldonado, A. (2010). Rol docente y asertividad. Tesis. Universidad Rafael Landívar facultad de humanidades. Quetzaltenango. Guatemala.

15. Marín, A. (Ed.). (2010). La nueva comunicación. Trotta. Madrid.

16. Méndez, C; (2009). Metodología. Diseño y desarrollo del proceso de investigación con énfasis en ciencias empresariales. 4ª Edición México.

17. Mosley, D. Pietro, L. y Megginson, P. (2005). Supervisión, La Práctica del Empowerment, Desarrollo de Equipos de trabajo y su Motivación (6ª . Edición). Editorial Thomson Editores. México. 
KOINONIA. Revista Arbitrada Interdisciplinaria de Ciencias de la Educación, Turismo, Ciencias Sociales y Económica, Ciencias del Agro y Mar y Ciencias Exactas y

18. Pelekais, C; Raspa, P y Pelekais, E. (2011). Hacia una cultura de investigación cualitativa. Ediciones Astro Data. S.A.

19.Pérez, A (2009) Comprender y transformar la enseñanza. Segunda ed. Madrid: Morata.

20.Pérez, D. (2010). Contribución de la historia y filosofía de las ciencias al desarrollo de un modelo de enseñanza/aprendizaje como investigación. Enseñanza de las Ciencias, 11(2), 197-212.

21.Piedra L. (2008). Estrategias didácticas. Facultad de Educación. Universidad de Costa Rica.

22.Pimienta, J. (2012) Estrategias de Enseñanza- Aprendizaje. México. Pearson Educación.

23. Roa, E. (2006) "Persona Humana Decente". Valores para una vida con sentido. Colección Semillas de Esperanza. Táchira, Venezuela.

24. Tamayo y Tamayo, M. (20012). Diccionario de la investigación científica. $3^{\circ}$ Edición. México. Limusa.

25. Tobón, S (2008) Estrategias didácticas para formar competencias. IV Congreso Internacional Virtual de Educación. Ciber educa.

(C2019 por el autor. Este artículo es de acceso abierto y distribuido según los términos y condiciones de la licencia Creative Commons Attribution (http://creativecommons.org/licenses/by/4.0/). 